\title{
Estilos parentales percibidos $y$ sintomatología depresiva en escolares de secundaria
}

\section{Marisol Ramírez-Caján ${ }^{1}$ \\ Giovani Díaz-Gervasi²}

RESUMEN: El objetivo de la investigación fue determinar la relación entre los estilos parentales percibidos y la sintomatología depresiva en escolares de secundaria en Lima. La muestra estuvo conformada por 281 escolares de 12 a 17 años de los cuales el $51.2 \%$ varones y el $48.8 \%$ mujeres. Para el análisis bivariado se evaluó la distribución de los datos a través del estadístico Kolgomorov-Smirnov y Kruskal-Wallis. Existe relación estadística entre los estilos parentales percibidos y la sintomatología depresiva. El $42.4 \%$ de la muestra presentó sintomatología depresiva. El estilo parental predominante fue el control sin afecto tanto en madres $(27.9 \%)$ como en padres (26.3\%). La sintomatología depresiva está relacionada a la edad y al sexo. La interacción de los padres e hijos es un factor determinante del estado emocional, pudiendo propiciar síntomas depresivos. Estos síntomas podrían ser confundidos con características psicosociales de la adolescencia.

\section{PALABRAS CLAVE: Depresión; Signos y Síntomas; Adolescente.}

Citar como: Ramírez M, Díaz G. Estilos parentales percibidos y sintomatología depresiva en escolares de secundaria. Un estudio comparativo. CASUS. 2017;2(2):119-126. 


\section{INTRODUCCIÓN}

La depresión es una de las principales causas de discapacidad en el mundo afectando a más de 350 millones de personas a nivel global (1). A este nivel se calcula que para el año 2020 la depresión será su segunda causa (1). Estudios nacionales revelan que el episodio depresivo en adolescentes cuenta con una prevalencia de $16.2 \%$, sin embargo dichas estadísticas varían según la cultura y ciudad. Así mismo, siendo la familia la unidad encargada de educar a sus hijos, es a través de los padres que se aprende una serie de actitudes, valores y comportamientos que permiten adaptarse a la sociedad (2). El 90\% de los adolescentes que cometen suicidio tiene como antecedente un diagnóstico de depresión y es más frecuente en mujeres (3). Hay dos mujeres deprimidas por cada hombre debido a causas psicofisiológicas en la adolescencia (4). Las mujeres presentan mayor depresión por los roles sexuales y tienden a ser más aprehensivas al momento de manejar el estrés, lo cual conlleva a la depresión (5).

Por otra parte, la familia es el principal soporte socioafectivo durante los primeros años de vida, por ello la importancia de identificar los factores de riesgo que pueden influenciar en la dinámica familiar y en problemas de salud mental. De esta manera, las patologías mentales, los estilos cognitivos y el clima emocional familiar son características parentales que podrían influir en el desarrollo de la depresión (6). La influencia de los padres hacia sus hijos es caracterizada por la cultura, la personalidad y la crianza. Los padres tienen la importante tarea de inculcar a sus hijos valores y normas, las cuales ayudaran al adolescente a enfrentarse a diversas adversidades.

Entre las actitudes más frecuentes en los adolescentes se encuentra la búsqueda de autonomía, de respeto y aceptación social. Estos deseos podrían contraponerse a los métodos de los padres para mantener el control y proteger a sus hijos. Además, el grupo par suele ejercer mayor influencia y es considerado una fuente de apoyo afectivo importante. Es importante conocer el comportamiento de estas variables en este grupo poblacional, debido a que algunos adolescentes atraviesan un conjunto de situaciones que pueden afectar su salud mental, dentro de estas es determinante el patrón de interacciones en la familia (7). Considerando lo anterior en el trabajo se indagó en la relación entre la sintomatología depresiva y los estilos parentales que perciben un grupo de adolescentes.

\section{MATERIALES Y MÉTODOS}

Estudio descriptivo correlacional de corte transversal. Se consideró una muestra de 281 escolares entre 12 y 17 años entre $2^{\circ}$ y $5^{\circ}$ de secundaria, de una institución educativa de Lima 144 eran varones y 137 mujeres. Se usó un muestreo no probabilístico.

Se utilizaron dos pruebas psicológicas para la medición de las variables principales. Los estilos parentales percibidos se definen como la percepción que tienen los hijos sobre los estilos y comportamientos de sus padres, variable politómica nominal categorizada en control sin afecto, afecto restrictivo, padre negligente y padre óptimo con el Parental Bonding Instrument (8); sintomatología depresiva definida como la sucesión de conductas y distorsiones cognitivas que pueden mantenerse a través del tiempo convirtiéndose en un trastorno depresivo (9) variable cuantitativa medida por el Inventario de Depresión de Beck II (9). Las variables sociodemográficas consideradas fueron: sexo, variable dicotómica (masculino, femenino); edad, variable continua y tipo de familia, variable nominal (extensa, nuclear y monoparental).

Se utilizó el programa estadístico SPSS versión 22 con el que se realizó un análisis exploratorio de los datos. A nivel descriptivo se consideraron frecuencias y porcentajes. Se evaluó la distribución de los datos a través del estadístico Kolgomorov-Smirnov. Luego se evaluó la correlación entre las variables principales para lo cual se utilizó la prueba de Kruskal-Wallis y Spearman. En la recolección de información se respetó la privacidad y el anonimato de los estudiantes. La investigación fue aprobada por un Comité de Ética.

\section{RESULTADOS}

E1 21\% de los encuestados presentó sintomatología depresiva leve, un $12.5 \%$ moderada y el $8.9 \%$ severa. El $42.4 \%$ de la muestra presentó sintomatología depresiva. Predominó el estilo parental control sin afecto para ambos sexos $(45.9 \%$ en madres y $47.3 \%$ en padres). Los varones representan el 51.2\% del total y la familia mayormente representada fue la extensa $43.1 \%$ (ver tabla 1$)$. 
Tabla 1. Características principales de los adolescentes

\begin{tabular}{lcc}
\hline & $\mathbf{n}$ & $\mathbf{( \% )}$ \\
\hline Sexo & & \\
Femenino & 137 & 48.8 \\
Masculino & 144 & 51.2 \\
& 14.4 & 1.4 \\
Edad (media + DS) & 111 & 39.5 \\
Tipo de familia & 121 & 43.1 \\
Nuclear & 49 & 17.4 \\
Extensa & & \\
Monoparental & & \\
& & \\
Sintomatología depresiva & 162 & 57.7 \\
Sin depresión & 59 & 21.0 \\
Leve & 35 & 12.5 \\
Moderado & 25 & 8.9 \\
Severo & & \\
& & \\
Estilos parentales (madres) & 19 & 6.8 \\
Padre óptimo & 59 & 21.0 \\
Padre negligente & 74 & 26.3 \\
Afecto restrictivo & 129 & 45.9 \\
Control sin afecto & & \\
Estilos parentales (padres) & & \\
Padre óptimo & 3 & 1.1 \\
Padre negligente & 123 & 43.8 \\
Afecto restrictivo & 22 & 7.8 \\
Control sin afecto & 133 & 47.3 \\
& & \\
\hline & & \\
\hline & & \\
& &
\end{tabular}

En la tabla 2 se evidencia que existe correlación estadística entre los estilos parentales percibidos y la sintomatología depresiva. Los valores medios más altos de sintomatología depresiva corresponden al estilo control $\sin$ afecto (media de la madre $=15.71$; media del padre $=15.56)$.

\section{Tabla 2 correlación entre estilos} parentales y sintomatología depresiva

\begin{tabular}{|c|c|c|c|}
\hline & \multicolumn{3}{|c|}{ Sintomatología depresiva } \\
\hline & media & ds & $p$-value \\
\hline $\begin{array}{l}\text { Tipos de estilos } \\
\text { parentales (madres) }\end{array}$ & & & 0.0001 \\
\hline Control sin afecto & 15.71 & 9.88 & \\
\hline Afecto restrictivo & 12.09 & 7.76 & \\
\hline Padre negligente & 13.68 & 10.22 & \\
\hline Padre óptimo & 6.84 & 4.51 & \\
\hline $\begin{array}{l}\text { Tipos de estilos } \\
\text { parentales (padres) }\end{array}$ & & & 0.0033 \\
\hline Control sin afecto & 15.56 & 9.58 & \\
\hline Afecto restrictivo & 10.54 & 10.26 & \\
\hline Padre negligente & 12.3 & 8.78 & \\
\hline Padre óptimo & 14.33 & 12.01 & \\
\hline
\end{tabular}

La correlación entre la edad y el sexo con la sintomatología depresiva es menor a 0.05 (ver tabla 3). 


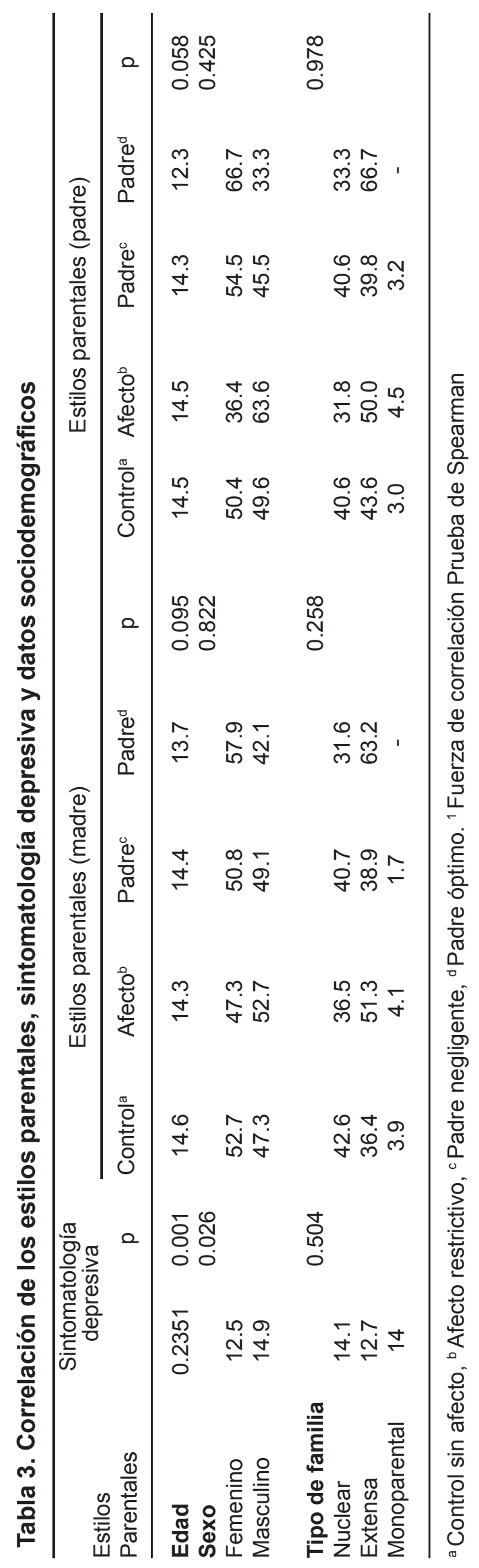




\section{DISCUSIÓN}

Existe relación significativa entre los estilos parentales percibidos y la sintomatología depresiva en los participantes. El $42.4 \%$ de la muestra presentó sintomatología depresiva. Por otra parte, el estilo control sin afecto en madres y padres es el más frecuente. La edad y el ser hombre se relacionan significativamente con la sintomatología depresiva.

Se evidencia que existe relación estadística entre el estilo parental percibido y la sintomatología depresiva en la muestra. Al respecto, un estudio refiere que el vínculo padre-hijo ha sido asociado a las respuestas cognitivas y afectivas de los jóvenes ante eventos vitales negativos y a la presencia de síntomas depresivos en la adolescencia (10). Aquellos que presentan un vínculo nocivo con sus padres son más vulnerables a presentar este tipo de síntomas sobre todo al enfrentar adversidades (11). Los resultados del presente estudio confirman la relación entre estilos parentales percibidos y el estado emocional de los hijos. Por lo tanto, la forma de vivenciar afectivamente las situaciones por parte de los adolescentes correspondería a la manera en que se comportan (incluyendo los estilos parentales) sus progenitores (12).

Se evidenció que el estilo control sin afecto es el más relacionado a la sintomatología depresiva. Este hallazgo podría estar vinculado a que los padres que ejercen un método rígido de corrección (violencia física, imposición de reglas), podrían afectar el estado emocional y causar síntomas depresivos en los adolescentes. Los padres que ejercen la labor educativa en el hogar a través del control excesivo de los comportamientos de sus hijos, podrían estar sujetos a más estrés. Este estilo podría predisponerlos a tomar decisiones rígidas o extremas sobre aspectos importantes del desarrollo del adolescente como son la búsqueda de libertad y autonomía, de expresar su propio punto de vista, de interactuar con diversos grupos sociales y con sus primeras experiencias afectivo-sexuales. La cohesión y flexibilidad que el adolescente percibe en su hogar son primordiales para tener un adecuado desarrollo emocional (7). Las familias con reglas poco flexibles, disciplina severa y excesivo control inducen a que el adolescente disminuya su autoestima, sintiéndose inseguro de asumir responsabilidades. Estos aspectos podrían desencadenar un trastorno depresivo (13).

Existe correlación significativa entre la edad, el sexo y la sintomatología depresiva. Los varones presentaron mayor sintomatología depresiva. El Estudio Epidemiológico Metropolitano de Salud Mental reportó una prevalencia de episodio depresivo en adolescentes de $8.6 \%$ en edades de 15 a 17 años con una mayor prevalencia en el sexo femenino $(14,15)$. Es necesario tener en cuenta que las creencias y costumbres de cada contexto son diversas, es por ello que el promedio de edad de los adolescentes podría variar de acuerdo al contexto biopsicosocial. Un estudio realizado en Colombia, sobre cognición, emoción y sintomatología depresiva en adolescentes escolarizados (16), halló también relación significativa entre las variables sintomatología depresiva y sexo. Se obtuvieron puntuaciones más altas en las mujeres que en los hombres. Así mismo, reportó que el rango de edad de mayor sintomatología depresiva se encuentra entre los 12 y los 16 años, lo que coincide con el presente estudio (17).

La mayor frecuencia de sintomatología depresiva en hombres reportada en este estudio, podría relacionarse con que los adolescentes probablemente no expresan sus sentimientos por temor y creencias machistas propias del entorno. Así mismo, la sintomatología depresiva en varones podría pasar desapercibida al ser confundida con cambios psicológicos propios de la adolescencia y a cierta resistencia frente a la búsqueda de ayuda psicológica para afrontar situaciones difíciles. Por otra parte, la manifestación emocional de tristeza y miedo son emociones más manifiestas en las mujeres, mientras que el enojo y la ira en los varones (18).

Así mismo, se espera que tanto mujeres como hombres actúen emocionalmente de manera diferente. El miedo y la tristeza son emociones que podrían estar sujetas a estereotipos sexuales (7). En el contexto latinoamericano es socialmente común pensar que expresar temor o tristeza profunda no es una conducta asociada al ser varón. Por lo tanto es posible que los hombres tiendan a no expresar sus emociones de manera espontánea e intensa, debido a que aprendieron que dentro del concepto de masculinidad, no es tan admisible sentir y demostrar miedo, tristeza, 
vergüenza o culpa (19). Esta condición podría disminuir la prevalencia detectada de la sintomatología depresiva en varones.

Los hallazgos del estudio podrían estar limitados ya que no obtuvo información de otras variables sociodemográficas de interés que hubiesen ayudado a una mayor comprensión del fenómeno. Por lo tanto, es importante considerar a futuro posibles variables vinculadas al estilo parental y a la sintomatología depresiva, tales como: los antecedentes depresivos familiares, aspectos socioeconómicos, número de hermanos y otros. Por otra parte, las respuestas en escalas de auto reporte pudieron ser influenciadas por la deseabilidad social.

\section{CONCLUSIONES}

El estilo parental percibido está relacionado a la sintomatología depresiva en los adolescentes estudiados. De esta manera la interacción de los padres e hijos es un factor determinante del estado emocional, pudiendo propiciar síntomas depresivos en los adolescentes. La sintomatología depresiva fue manifestada por un $42.4 \%$ de la muestra, lo que significa que puede ser una situación más frecuente de lo que se cree y para la cual aún no se desarrollan campañas preventivas.
El estilo parental predominante tanto en madres y padres es el control sin afecto el cual estaría caracterizado por presentar poca atención a las necesidades de los hijos, tener actitudes controladoras hacia su comportamiento y manifestar un trato vertical, planteando reglas rígidas en la dinámica familiar. Este estilo parental puede desencadenar síntomas depresivos en una etapa vulnerable como la adolescencia.

Se aprecia que la edad y el sexo son factores relacionados a la sintomatología depresiva en la adolescencia. Los estilos parentales percibidos por el adolescente podrían exponerlo a síntomas depresivos. Atípicamente, en el presente estudio los hombres presentaron mayor sintomatología depresiva, lo cual puede estar relacionado a factores socioculturales. No obstante, la depresión en ambos sexos es un fenómeno multicausal, por lo tanto, las diferencias entre mujeres y hombres carecen de explicación exacta.

Se sugiere promover la intervención psicológica preventiva a nivel comunitario y escolar dirigida a adolescentes y padres de familia sobre estilos parentales adecuados y cómo detectar y afrontar en familia los síntomas depresivos. En estudios futuros sería viable evaluar la autopercepción de los padres ante la crianza de sus hijos.

\section{REFERENCIAS BIBLIOGRÁFICAS}

1. Marcus M, Yasamy T, Ommeren M, Chisholm D, Saxena S. La depresión una preocupación de la salud publica global. Federación Mundial de la Salud Mental; 2012.

2. Ministerio de educación, cultura y deporte. La participación de las familias en la educación escolar. 2014.

3. Puskar K, Sereika S, Tusaie K. Effect of the teaching kids to cope program on outcomes of depression and coping among rural adolescents. Journal of Child and Adolescent Psychiatric Nursing. 2003;2(1):71-80.

4. Steinberg L. Una perspectiva de la neurociencia social sobre la toma de riesgo de los adolescentes. Dev Rev. 2008;28(1):78-106.

5. Foster J, Kuperminc G, Price A. Gender differences in posttraumatic stress and related symptoms among
6. inner-city youth exposed to community violence. Journal of Youth and Adolescence. 2004;33(1):59-69.

7. Sander J, Mc Carty C. Youth depression in the family context: Familial risk factors and models of treatment. Clin Child Fam Psychol Rev. 2005;8(3):203-219.

8. Oliva A. Relaciones familiares y desarrollo en adolescentes. Anuario de Psicología. 2006;37(3):209-223.

9. Amézquita M. Validez de constructo del Parental Bonding Instrument en una muestra de adolescentes de 5to de secundaria de un colegio público de Lima metropolitana [tesis]. Lima: Pontificia Universidad Católica del Perú. Facultad de Ciencias y Letras Humanas; 2013.

10. Alcantara N. Sintomatología depresiva y adhesión al tratamiento en pacientes con VIH [tesis]. Lima:
11. Pontificia Universidad Católica del Perú. Facultad de Ciencias y Letras Humanas; 2008.

12. Martínez A, Muñoz A. Modelos explicativos sobre la depresión y el malestar emocional entre los adolescentes barceloneses. Revista de Salud Mental. 2010;33(2):145-152.

13. Kraaij V, Garnefski N, Wilde E, Dijkstra A. Negative life events and depressive symptoms in late adolescence: Bonding and cognitive coping as vulnerability factors Journal of Youth and Adolescence. Journal of youth and adolescence. 2003;32(3):185-193.

14. Rice F. Desarrollo Humano estudio del ciclo vital. México D.F.: Prentice-Hall Hispanoamericana; 1997. 
15. Álvarez M, Zúñiga B, Ramírez J, Silva A, Rodríguez $\mathrm{N}$, et al. La relación entre depresión y conflictos familiares en adolescentes. International Journal of Psychology and Psychological Therapy. 2009;9(2):205-216.

16. Instituto Nacional de Salud Mental. Estudio epidemiológico de salud mental en Lima Metropolitana y el Callao 2012. Lima: Instituto Nacional de Salud Mental; 2013.
17. Vargas H, Valverde J. Prevalencia y factores asociados con el episodio depresivo en adolescentes de Lima Metropolitana y Callao. Revista Peruana de Epidemiologia. 2010;14(2):91-98.

18. Yvonne G. Cognición, emoción y sintomatología depresiva en adolescentes escolarizados. Revista Latinoamericana de Psicología. 2007;39(3):435-447.
19. Nolen S, Girgus J. The emergence of gender differences in depression during adolescence. Psycho Bull. 1994;115(2):424-443

20. Hutson-Comeaux S, Kelly J. Gender stereotypes of emotional reactions: how we judge a emotion as valid. Sex Roles. 2002;47(1):1-10.

21. Condry J and Condry S. Sex differences: A study of the eye of the beholder. Child Development. 1976;47(2):812-819. 


\section{Perceived parenting styles and depressive symptomatology in high school students}

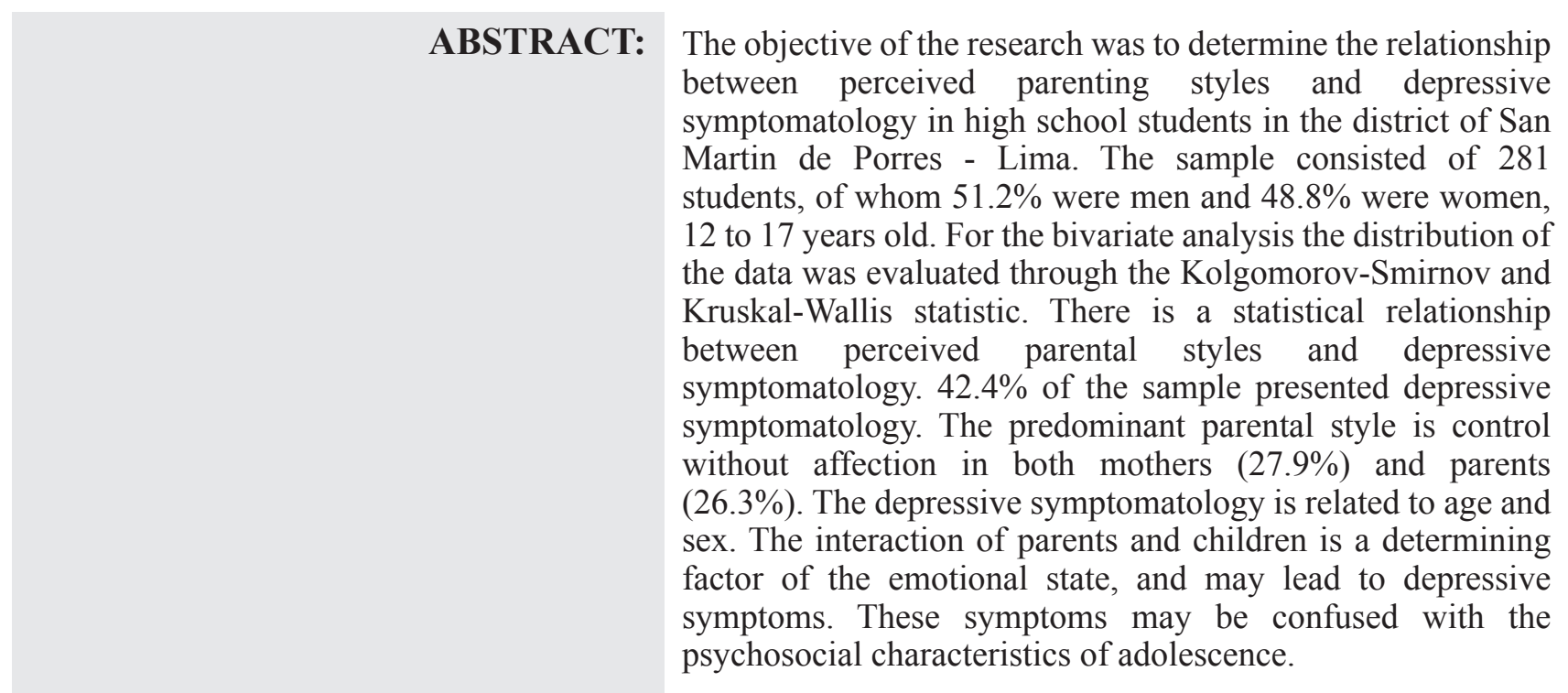

KEY WORDS: Depression; Signs and Symptoms; Adolescent. 